\title{
Uso do laser de baixa potência em estrias de distensão: ensaio clínico randomizado controlado
}

\author{
Use of low level laser therapy in striae distensae: a randomized controlled trial \\ Bethina Bernardon Busatta ${ }^{1}$, Karoline Calichio Medeiro' ${ }^{1}$, Lorrainy Rufino Velozo' ${ }^{1}$ Camila Mayumi Martin Kakihata ${ }^{1}$, \\ Francyelle dos Santos Soares ${ }^{1}$, Márcia Rosângela Buzanello Azevedo', Gladson Ricardo Flor Bertolini ${ }^{1,2} \square$
}

' Colegiado de Fisioterapia da Universidade Estadual do Oeste do Paraná (Unioeste). Cascavel, PR.

2 Programa de Pós-Graduação em Biociências e Saúde da Unioeste. Cascavel, PR.

Como citar este artigo (How to cite this article):

Busatta BB, Medeiro KC, Velozo LR, Kakihata CMM, Soares FS, Azevedo MRB, Bertolini GRF. Uso do laser de baixa potência em estrias de distensão: ensaio clínico randomizado controlado (Use of low level laser therapy in striae distensae: a randomized controlled trial). Sci Med. 2018;28(2):ID28710. http://doi.org/10.15448/1980-6108.2018.2.28710

\section{RESUMO}

OBJETIVOS: Avaliar os efeitos da laserterapia de baixa potência $(660 \mathrm{~nm})$ no tratamento de estrias albas.

MÉTODOS: Foi realizado um ensaio clínico randomizado com 20 mulheres divididas por igual em grupo controle e grupo tratamento. Para inclusão elas deveriam possuir estrias albas em região glútea e aceitar participar do estudo. Os critérios de exclusão foram: realização de outro tratamento para estrias, estado gravídico, uso de esteroides, tabagismo, diagnóstico de câncer, áreas hemorrágicas na pele, diagnóstico de epilepsia, diagnóstico de trombose, ou qualquer alteração cognitiva. Foram realizadas 12 aplicações de laser $660 \mathrm{~nm}$, durante quatro semanas. Após mais quatro semanas de seguimento, foi realizada uma reavaliação. Após cada aplicação foram feitas fotos da região para avaliação do tamanho das estrias por meio de planimetria digitalizada, que avalia a porcentagem de área ocupada pelas estrias. Para essa avaliação foram utilizadas as fotos por ocasião da primeira aplicação (AV1), da sexta (AV2), da 12a (AV3) e da última avaliação, após quatro semanas do término do tratamento (AV4). Foram realizadas também avaliações qualitativas por tato (profundidade) e inspeção visual (tonalidade e tamanho).

RESULTADOS: A análise pela planimetria demonstrou que não houve diferença entre a área ocupada pelas estrias quando se compararam as diversas avaliações do mesmo grupo, até a AV3. Porém na AV4 (quatro semanas após a última aplicação de laser) do grupo tratamento, houve diminuição estatisticamente significativa da área de estrias em relação à AV1. No grupo controle, não houve diferença entre AV4 e as outras avaliações. Na comparação entre os dois grupos, não houve diferença nas três primeiras avaliações. Porém na AV4 o grupo tratamento mostrou menor área das estrias em relação ao grupo controle. Na análise qualitativa o grupo tratado apresentou menor visibilidade e menor profundidade das estrias ao tato.

CONCLUSÕES: A terapia com 12 sessões de laser de baixa potência durante quatro semanas teve efeito positivo no tratamento de estrias albas, com os resultados tornando-se evidentes somente quatro semanas após a última aplicação. O laser de $660 \mathrm{~nm}$ mostrou-se um método não invasivo, indolor e de rápida aplicação.

DESCRITORES: estrias de distensão; terapia com luz de baixa intensidade; terapia a laser.

\section{ABSTRACT}

AIMS: To analyze the effects of low-level laser therapy $(660 \mathrm{~nm})$ in the treatment of striae alba.

METHODS: A randomized clinical trial was conducted with 20 women equally divided into a control group and a treatment group. For inclusion, they should have striae alba in the gluteal region and accept to participate in the study. Exclusion criteria were other treatment for striae, pregnancy, steroid use, smoking, cancer diagnosis, hemorrhagic areas on the skin, diagnosis of epilepsy, diagnosis of thrombosis, or any cognitive impairment. Twelve applications of $660 \mathrm{~nm}$ laser were performed for four weeks. After another four weeks of follow-up, a reevaluation was performed. After each application, photos were taken from the region to evaluate the size of the striae by means of scanned planimetry, which calculates the percentage of area occupied by the striae. For this evaluation, the photos taken at the first application (AV1), the sixth (AV2), the 12th (AV3) and the last evaluation, after four weeks of treatment completion (AV4), were used. Qualitative evaluations were also performed by touch (depth) and visual inspection (hue and size).

RESULTS: The analysis by planimetry showed that there was no difference between the area occupied by the striae when comparing the various evaluations of the same group, until the AV3. However in AV4 (four weeks after the last laser application) of the treatment group, there was a statistically significant decrease of the stria area in relation to AV1. In the control group, there was no difference between AV4 and the other evaluations. In the comparison between the two groups, there was no difference in the first three evaluations. However in AV4 the treatment group showed a smaller area of the striae in relation to the control group. In the qualitative analysis the treated group had lower visibility and less depth of the striae to the touch.

CONCLUSIONS: Therapy with 12 sessions of low-level laser for four weeks had a positive effect on the treatment of striae alba, with results becoming evident only four weeks after the last application. The $660 \mathrm{~nm}$ laser showed to be a non-invasive, painless and fast-applied method. KEYWORDS: striae distensae; low-level light therapy; laser therapy. 
Abreviaturas: ATP, adenosina trifosfato; AV1, primeira avaliação; AV2, segunda avaliação; AV3, terceira avaliação; AV4, quarta avaliação; IMC, índice de massa corporal.

\section{INTRODUÇÃO}

Estrias de distensão são alterações cutâneas que acometem grande parte da população, principalmente mulheres. São caracterizadas por uma lesão no tecido conjuntivo dérmico com consequente diminuição dos elementos da pele. Embora não estejam ligadas diretamente a questões de saúde, essas lesões podem causar desconforto, trazendo problemas de ordem estética e até psicológica. Por esses motivos vem aumentando a busca por tratamentos cada vez mais eficazes [1].

As estrias aparecem mais frequentemente nas mamas, abdômen, nádegas e coxa, dependendo da circunstância em que se desenvolveram. Tem sido relatada na literatura prevalência de $40 \%$ a $70 \%$ em adolescentes e de $90 \%$ após a gestação. As taxas de prevalência das estrias, a idade de início e o predomínio em determinado sexo dependem da população estudada [2].

A primeira descrição morfológica das estrias de distensão ocorreu em 1936, sendo essas lesões nomeadas de estrias atróficas. Duas formas clínicas e histopatologicamente reconhecíveis foram descritas: estrias rubras e estrias albas. As lesões, inicialmente eritematosas e arroxeadas, são referidas como estrias rubras; com o passar do tempo tornam-se enrugadas e hipopigmentadas, formando marcas atróficas como cicatrizes, sendo então denominadas estrias albas. Estas têm sido descritas como uma forma permanente de estria [3]. Segundo Monteiro et al. [4], as causas das estrias não são claras. Uma série de teorias têm sido propostas, incluindo infecção, efeito mecânico de alongamento (como na obesidade e na gravidez), aumento de hormônios esteroides, fatores genéticos, estados de imunossupressão e doenças crônicas do fígado.

Para Salter et al. [5], independentemente da etiologia, todas as estrias seguem uma progressão. Biópsias comparando áreas com estrias e pele normal mostram reorganização e diminuição da rede de fibras elásticas na derme. As lesões causadas por estrias exibem características semelhantes às das cicatrizes. Histopatologicamente, apresentam-se como dano cicatricial no colágeno dérmico, tecido elástico e matriz extracelular [2].
Vários tratamentos têm sido propostos e estudados. Dentre eles destacam-se a laserterapia e outros dispositivos de luz que estimulam produção de colágeno e elastina; aplicação de produtos tópicos que segundo alguns estudos estimulam regeneração fibroelástica; microdermoabrasão, que induz a alterações epidérmicas promovendo remodelação da matriz; além da terapia com radiofrequência, que promove a contração de fibrilas e induz à formação de colágeno [3].

O laser de baixa potência é empregado para acelerar e melhorar a qualidade do processo regenerativo. Esse recurso acelera o processo de reparo, atuando na sequência de eventos fisiológicos e bioquímicos decorrentes do processo, como a inflamação, a síntese de colágeno, a formação do tecido de granulação e a reepitelização [6-9]. A energia absorvida é transformada em adenosina trifosfato (ATP) e usada pela célula para atividades metabólicas, como proliferação celular, síntese de colágeno e aceleração do processo de reparação do tecido em animais e humanos [9]. Os lasers em baixa intensidade não possuem potencial destrutivo e apresentam eficiente ação anti-inflamatória e analgésica. A camada tecidual a ser atingida depende do tipo de laser, da potência, do comprimento de onda e do tempo de irradiação [11].

Visto que as estrias albas podem ter impacto estético e psicológico, e que o laser terapêutico representa, de forma potencial, um recurso terapêutico valioso para o seu tratamento, este estudo teve como objetivo analisar os efeitos da laserterapia de baixa potência $(660 \mathrm{~nm})$ no tratamento de estrias albas em região glútea de mulheres jovens.

\section{MÉTODOS}

\section{Delineamento, amostra e aspectos éticos}

Foi realizado um estudo do tipo ensaio clínico randomizado, entre julho e dezembro de 2016, no Centro de Reabilitação Física da Universidade Estadual do Oeste do Paraná (Unioeste), na cidade de Cascavel, PR. A pesquisa foi aprovada pelo Comitê de Ética em Pesquisa da Unioeste (parecer consubstanciado 1.621.030). As participantes da pesquisa foram 20 mulheres, estudantes da Unioeste, com idade entre 18 e 22 anos, que possuíam estrias albas em região glútea. Todas aceitaram participar voluntariamente da pesquisa e assinaram o Termo de Consentimento Livre e Esclarecido antes do início do tratamento. Os critérios de exclusão foram: 1) estar realizando ou já ter realizado outro tratamento para estrias; 2) estado gravídico; 3 ) uso de medicamentos esteroides; 
4) tabagismo; 5) diagnóstico de câncer; 6) presença de áreas hemorrágicas na pele; 7) diagnóstico de epilepsia ou trombose; e 8) alteração cognitiva.

A amostra foi recrutada por convite verbal e cartazes. Após descartada a presença de critérios de exclusão, as voluntárias foram randomizadas, por meio de sorteio em envelope opaco, para a alocação no grupos controle e tratamento, cada um deles contendo 10 participantes, sempre pelo mesmo pesquisador. Durante a aplicação do laser elas foram orientadas a não realizar nenhum outro tipo de tratamento para estrias albas, nem uso de medicamentos fotossensibilizantes. Dados referentes à idade e cor da pele das participantes foram obtidos a partir de um questionário autorreferido, preenchido no início da pesquisa.

\section{Protocolo de tratamento}

A aplicação do laser ocorreu durante quatro semanas, sendo realizadas três aplicações semanais, totalizando 12 aplicações. O laser usado foi de diodo com comprimento de onda de $660 \mathrm{~nm}$ (potência de $30 \mathrm{~mW}$ ) e densidade de energia de $4 \mathrm{~J} / \mathrm{cm}^{2}$, com energia total (diária) de 2,16 J (Laserpulse, Ibramed ${ }^{\circledR}$. Amparo, Brasil). A aplicação foi pontual, em contato direto com a pele, com uma distância de $1 \mathrm{~cm}$ por ponto dentro de uma região de $3 \mathrm{~cm}$ por $3 \mathrm{~cm}$, totalizando nove pontos, com duração de oito segundos por ponto. Previamente ao início de seu uso o equipamento foi aferido com relação à potência de emissão.

Para as aplicações as voluntárias deveriam permanecer em decúbito ventral em uma maca, com a região a ser tratada livre de peças de roupas. A região era higienizada com algodão embebido em álcool $70 \%$. Para a marcação dos pontos utilizou-se uma folha transparente de tamanho A4, a qual continha as marcações das áreas de $3 \mathrm{~cm}$ por $3 \mathrm{~cm}$, com a área dentro dos pontos aberta, para permitir o contato direto entre a caneta e a pele da voluntária. A folha A4 era posicionada na lateral do glúteo em que seria aplicado o laser, tendo como referência para posicionamento o osso sacro e a borda superior da crista ilíaca. As pacientes colocavam os óculos para proteção contra o laser e então realizava-se a aplicação do mesmo.

No grupo controle foi realizada terapia do tipo placebo, em que foi simulada a aplicação com os procedimentos semelhantes aos realizados no grupo tratamento, encostando a caneta do aparelho sobre a pele da voluntária, porém sem emissão de laser.

\section{Avaliações}

As voluntárias tiveram seu peso verificado antes do início das aplicações do laser e no dia da última avaliação, para descartar a possibilidade de influência do ganho ou de perda de peso sobre o aspecto da pele. Foi calculado o índice de massa corporal (IMC) para comparação entre o grupo tratamento e o grupo controle.

Antes de iniciar o tratamento, e após cada aplicação, foram feitas fotos da região para avaliação do tamanho das estrias por meio de planimetria digitalizada. Quatro semanas após a 12 ${ }^{\mathrm{a}}$ aplicação foi feita a última avaliação. Para a análise foram utilizadas as fotos obtidas na avaliação inicial, antes de iniciar o tratamento (AV1), após seis aplicações (AV2), após 12 aplicações (AV3) e após quatro semanas de seguimento (AV4). Os procedimentos de avaliação foram iguais para o grupo tratamento e o grupo controle.

Visando expor a área de pele a ser fotografada, a voluntária era posicionada em decúbito lateral, e uma estrutura de acrílico formada por duas laterais com aproximadamente $40 \mathrm{~cm}$ de altura e um teto com abertura de $7 \mathrm{~cm} \times 7 \mathrm{~cm}$ era apoiada na maca sobre a região a ser realizada a terapia. A câmera fotográfica $\left(\right.$ Samsung $^{\circledR}$ ), com resolução de $3264 \times 2448$ pixels, era posicionada na abertura e contava-se com a ajuda de uma luz artificial posicionada lateralmente à estrutura, de forma que iluminasse a região fotografada sem produzir reflexos.

Para análise quantitativa do tamanho das estrias, utilizou-se o programa Image-Pro Plus ${ }^{\circledR}$, que por meio de planimetria avalia a área ocupada pelas estrias nas fotos, com sobreposição de uma grade contendo 150 pontos. Contando-se o número de pontos de intersecção ocupados pelas estrias e aplicando-se a seguinte fórmula: número de pontos $\times 100 / 150$ (número total de pontos da grade) obteve-se a porcentagem de área ocupada pelas estrias. Essa avaliação foi realizada sempre pelo mesmo examinador. Os resultados obtidos em cada grupo foram expressos em média e desvio padrão.

Também foi realizada uma análise qualitativa, nos mesmos dias em que foram realizadas as capturas de imagem. $\mathrm{O}$ mesmo examinador avaliou a profundidade das estrias ao tato, bem como a tonalidade e o tamanho das mesmas por inspeção visual.

\section{Análise estatística}

Os resultados foram tabelados no Programa Excel 15.0 $\left(\right.$ Microsoft $\left.^{\mathbb{R}}\right)$ e, para os cálculos, foi utilizado o 
programa Bioestat 5.0. Após o teste de Shapiro-Wilk foi feita análise de variância unidirecional com pósteste de Bonferroni para comparações intragupo. Para comparações intergrupo, nas diferentes avaliações, foi utilizado o teste t de Student não pareado. Em todos os casos o nível de significância foi de $5 \%$. Tendo como base a amostra de 10 voluntárias por grupo, o desvio-padrão de 5,9 com diferença a ser detectada de 6,4 e nível de significância de $5 \%$, o poder do teste foi de $80 \%$.

\section{RESULTADOS}

Não houve perda amostral dentre as 20 voluntárias recrutadas para o estudo. Na Tabela 1 observa-se que os dois grupos (tratamento e controle) foram homogêneos em idade, IMC e cor da pele.

A análise pela planimetria demonstrou que não houve diferença entre a área ocupada pelas estrias quando se compararam as diversas avaliações do mesmo grupo, até a AV3. Porém na AV4 (quatro semanas após a última aplicação de laser) do grupo tratamento, houve diminuição estatisticamente significativa da área de estrias em relação à AV1. No grupo controle, não houve diferença entre AV4 e as outras avaliações (Tabela 2).
Na comparação entre os dois grupos, não houve diferença nas três primeiras avaliações. Porém na AV4 o grupo tratamento mostrou redução da área das estrias em relação ao grupo controle (Tabela 2).

$\mathrm{Na}$ análise qualitativa foi possível observar alteração na profundidade das estrias ao tato, pois as mesmas tornaram-se menos profundas e a pele mais homogênea. A tonalidade das estrias também mudou, tornando-as menos visíveis. Percebeu-se redução do tamanho das estrias, e em amostras que possuíam estrias mais finas notou-se visualmente maior progresso em direção ao desaparecimento das mesmas. A Figura 1 mostra o aspecto no momento da AV1 e da AV4, para ambos os grupos. Não foram observados efeitos colaterais, como edema, eritema ou dor.

Tabela 1. Características das 20 voluntárias, randomizadas para os dois grupos.

\begin{tabular}{lccc}
\hline & $\begin{array}{c}\text { Grupo Controle } \\
\text { Média } \pm \text { DP }\end{array}$ & $\begin{array}{c}\text { Grupo Tratamento } \\
\text { Média } \pm \text { DP }\end{array}$ & p \\
\hline Peso $(\mathrm{kg})$ & $59,2 \pm 7,2$ & $58,9 \pm 11,4$ & 0,7045 \\
Índice da massa corporal $\left(\mathrm{kg} / \mathrm{m}^{2}\right)$ & $22,4 \pm 2,8$ & $21,1 \pm 3,1$ & 0,3943 \\
Idade (anos) & $20,0 \pm 0,9$ & $19,8 \pm 2,0$ & 0,6854 \\
Pele branca $(\mathrm{n})$ & 8 voluntárias & 7 voluntárias & \\
Pele parda $(\mathrm{n})$ & 2 voluntárias & 3 voluntárias & \\
\hline
\end{tabular}

Tabela 2. Resultados da avaliação por planimetria digitalizada realizada em estrias albas de 20 voluntárias tratadas com laser de baixa potência, randomizadas para Grupo Controle e Grupo Tratamento.

\begin{tabular}{lccccc}
\hline & \multicolumn{5}{c}{ Porcentagem de área ocupada pelas estrias } \\
\cline { 2 - 5 } Grupo & AV1 & AV2 & AV3 & AV4 & p-valor \\
\cline { 2 - 5 } & Média \pm DP & Média \pm DP & Média \pm DP & Média \pm DP & \\
Grupo Controle & $23,95 \pm 5,92$ & $23,73 \pm 6,22$ & $24,48 \pm 6,30$ & $24,70 \pm 5,67$ & 0,9817 \\
Grupo Tratamento & $26,71 \pm 7,58^{*}$ & $22,95 \pm 5,50$ & $20,41 \pm 5,57$ & $18,30 \pm 5,01$ & $\mathbf{0 , 0 2 1 3}^{*}$ \\
p-valor & 0,1887 & 0,3857 & 0,0716 & $\mathbf{0 , 0 0 7 7 +}^{+}$ & \\
\hline
\end{tabular}

AV1, avaliação antes do início do tratamento; AV2, avaliação após 6 aplicações; AV3, avaliação após 12 aplicações; AV4, avaliação 4 semanas após a última aplicação; DP, desvio padrão.

* Diferença entre AV1 e AV4 para o grupo tratamento. ANOVA unidirecional com pós-teste de Bonferroni.

+ Diferença entre grupo tratamento e grupo controle. Teste t não pareado. 

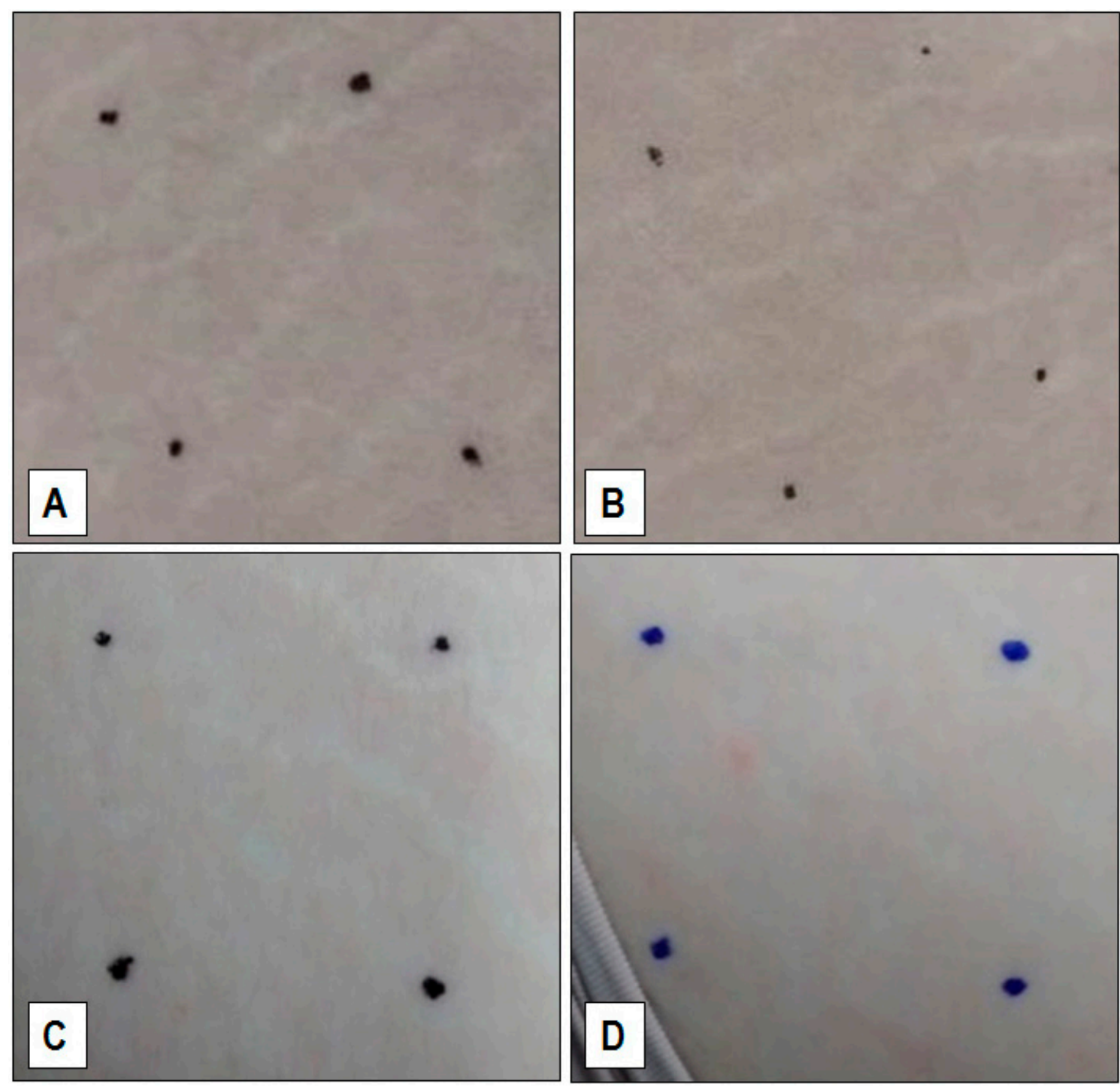

Figura 1. Fotografias representativas do grupo controle e do grupo tratamento, mostrando evolução ao longo do tempo para o grupo tratado, no qual as estrias tornaram-se menos profundas e visíveis. A e B: voluntária do grupo controle; B e C: voluntária do grupo tratamento; ambas com 19 anos de idade, com estrias albas na região glútea. A e C: antes do tratamento; B e D: após 4 semanas do término do tratamento.

\section{DISCUSSÃO}

Observou-se neste estudo que houve melhora das estrias albas após quatro semanas do término das 12 aplicações de laser de baixa potência (comprimento de onda de $660 \mathrm{~nm}$ e potência de $30 \mathrm{~mW}$ ). A laserterapia de baixa intensidade já é reconhecida como um método de tratamento eficaz para cicatrização de tecidos. Vários estudos in vitro e in vivo têm mostrado ações positivas na perfusão tecidual e estimulo à neovascularização, proliferação fibroblástica e queratinócitos, aumento na síntese e deposição de colágeno e hidroxiprolina, diminuição de mediadores inflamatórios como IL-1 $\beta$,
IL-10, TNF- $\alpha$ e NF- $\kappa B$, diminuição do estresse oxidativo e aceleração da cura tecidual [7,9, 12-15].

$\mathrm{Na}$ literatura publicada encontram-se alguns estudos relacionados ao uso do laser em estrias, contudo, tanto os comprimentos de onda, quanto a potência dos equipamentos são diferentes daqueles utilizados na terapia com laser de baixa potência [16-21]. Como no estudo de Tay et al. [21], que utilizaram o laser fracionado não ablativo de $1540 \mathrm{~nm}$ em estrias atróficas, porém não observaram qualquer melhora nas estrias tratadas, apenas eritema e hiperpigmentação pósinflamatória. De forma diferente, Angelis et al. [16], que também utilizaram o laser de érbium fracionado 
não ablativo de $1540 \mathrm{~nm}$, observaram espessamento de epiderme e derme e nova deposição de fibras colágenas e elásticas um mês após o tratamento, relatando que observaram como efeitos colaterais eritema e edema, o que não ocorreu no presente estudo, provavelmente por tratar-se de um laser de baixa potência.

Para equipamentos laser do tipo excimer, Ostovari et al. [19] utilizaram o comprimento de onda de $308 \mathrm{~nm}$ e relatam, com base em medidas e pela qualidade observada em fotografias, que o laser foi pouco efetivo na repigmentação das estrias albas, com a maior parte dos pacientes (80\%), obtendo resultados considerados fracos ou moderados. Já Goldberg et al. [17] aplicaram o laser ultravioleta $\mathrm{B}$, tendo como respostas aumento do conteúdo de melanina e hipertrofia e aumento do número de melanócitos. Todos esses efeitos, apesar de não terem sido avaliados no presente estudo, podem ter ocorrido, visto que houve alteração na tonalidade das estrias albas, que ficaram com coloração mais próxima da pele.

$\mathrm{O}$ laser de $\mathrm{CO}_{2}$ também é considerado uma opção terapêutica em casos de estrias, sendo que Lee et al. [18] utilizando esse tipo de laser com $10.600 \mathrm{~nm}$ observaram efeitos positivos, tanto melhora clínica quanto satisfação dos pacientes com o tratamento. Shin et al. [20] também observaram resultados positivos clinicamente, contudo, em biópsias não houve maior deposição de colágeno em comparação ao grupo placebo. No estudo de Ryu et al. [22] constatou-se que as biópsias do pós tratamento não apresentaram aumento da espessura epidérmica, mas houve aumento na quantidade de colágeno e as fibras elásticas sofreram alteração, com novas fibras surgindo na derme superior. Yang e Lee [23] também observaram melhora clínica e histológica, com aumento da espessura da epiderme e das fibras colágenas e elásticas na derme. Essas terapias também não são isentas de efeitos colaterais, podendo produzir eritema e hiperpigmentação [21].

Dados publicados sobre o tratamento da pele com laser de baixa potência mostram que ele promove o reparo de feridas na pele [24], produzindo neovascularização, diminuição de infiltrado inflamatório, proliferação fibroblástica e consequente aumento do conteúdo colágeno; e, além disso, produzem menos efeitos colaterais [25-27]. Esses dados serviram de base para a escolha do comprimento de onda de $660 \mathrm{~nm}$, com potência de $30 \mathrm{~mW}$, na realização do presente estudo.
A constatação de que o efeito do tratamento com o laser de baixa potência só foi perceptível após quatro semanas da última aplicação tem uma importante implicação prática. Os pacientes precisam ser informados de que existe esse período de latência até que o resultado possa ser observado.

Como limitações deste estudo podemos considerar que a avaliação qualitativa, bastante utilizada em estudos desta natureza [16-21,28], poderia ter sido feita de forma cega. Entretanto, além da avaliação qualitativa, buscou-se analisar quantitativamente as alterações nas estrias por meio da planimetria, sendo esta uma das formas de avaliação via imagem [28]. Assim, pode-se inferir objetivamente que os resultados obtidos, de diminuição dos pontos de intersecção, indicam redução das estrias. A ausência de análise por biópsia também foi uma limitação do estudo, contudo, os resultados observados, mesmo que indiretamente, indicam que ocorreram os efeitos apontados pelos estudos anteriores que utilizaram a análise anatomopatológica.

Mesmo considerando as limitações, este estudo permite concluir que a terapia com laser de baixa potência, em três aplicações semanais por quatro semanas, apresentou efeito positivo no tratamento de estrias albas, o qual somente foi observado após quatro semanas do término do tratamento. Além de eficaz, a laserterapia de baixa potência constituiu-se em um método não invasivo, indolor e de rápida aplicação.

\section{NOTAS}

Apoio financeiro

Este estudo não recebeu apoio financeiro de fontes externas.

Declaração de conflito de interesses

Os autores declaram não haver conflitos de interesses relevantes ao conteúdo deste estudo.

\section{Contribuições dos autores}

Todos os autores fizeram contribuições substanciais para concepção, ou delineamento, ou aquisição, ou análise ou interpretação de dados; e redação do trabalho ou revisão crítica; e aprovação final da versão para publicação.

Disponibilidade dos dados

Os autores declaram ter tido acesso a todos os dados obtidos e assumem completa responsabilidade pela integridade destes resultados. 


\section{REFERÊNCIAS}

1. Rezende PP, Pinheiro NM, Mendonça AC. Recursos terapêuticos para tratamento de estrias de distensão: uma revisão sistemática. JCBS. 2016;1(3):59-67.

2. Sato MS, Silva LLG da, Brenner FM, Tung R. Avaliação histológica comparativa da eficácia de ácido tricloroacético e subcisão, isolados e combinados, no tratamento de estrias abdominais. Surg Cosmet Dermatol. 2012;4(1):31-7.

3. Elsaie ML, Baumann LS, Elsaaiee LT. Striae distensae (Stretch Marks) and different modalities of therapy: An update. Dermatologic Surg. 2009;35(4):563-73. https://doi.org/10.1111/j.1524-4725.2009.01094.x

4. Guimarães PAMP, Haddad A, Sabino Neto M, Lage FC, Ferreira LM. Striae distensae after breast augmentation: treatment using the nonablative fractionated 1550-nm Erbium Glass Laser. Plast Reconstr Surg. 2013;131(3):636-42. https://doi. org/10.1097/PRS.0b013e31827c7010

5. Salter SA, Kimball AB. Striae gravidarum. Clin Dermatol. 2006;24(2):97-100. https://doi.org/10.1016/j.clindermatol. 2005.10.008

6. Freitas RPA, Barcelos APM, Nóbrega BM, Macedo AB, Oliveira AR, Ramos AMO, Vieira WHB. Laserterapia e microcorrente na cicatrização de queimadura em ratos. Terapias associadas ou isoladas? Fisioter e Pesqui. 2013;20(1): 24-30. https://doi.org/10.1590/S1809-29502013000100005

7. Dahmardehei M, Kazemikhoo N, Vaghardoost R, Mokmeli S, Momeni M, Nilforoushzadeh MA, Ansari F, Amirkhani A. Effects of low level laser therapy on the prognosis of split-thickness skin graft in type 3 burn of diabetic patients: a case series. Lasers Med Sci. 2016;31(3):497-502. https://doi.org/10.1007/s10103-016-1896-9

8. Dancákova L, Vasilenko T, Kovác I, Jakubcova K, Holly M, Revajova V, Sabol F, Tomori Z, Iversen M, Gál P, PhD, Bjordal JM. Low-level laser therapy with $810 \mathrm{~nm}$ wavelength improves skin wound healing in rats. Photomed Laser Surg. 2014;32(4):198-204. https://doi.org/10.1089/pho.2013.3586

9. Gupta A, Keshri GK, Yadav A, Gola S, Chauhan S, Salhan AK, Singh SB. Superpulsed (Ga-As, 904 nm) low-level laser therapy (LLLT) attenuates inflammatory response and enhances healing of burn wounds. J Biophotonics. 2015;8(6): 489-501. https://doi.org/10.1002/jbio.201400058

10. Leite SN, Andrade TAM de, Masson-Meyers D dos S, Leite MN, Enwemeka CS, Frade MAC. Phototherapy promotes healing of cutaneous wounds in undernourished rats. An Bras Dermatol. 2014;89(6):899-904. https://doi.org/10.1590/ abd1806-4841.20143356

11. Leal CT, Bezerra A de L, Lemos A. A efetividade do laser de HeNe 632,8 nm no reestabelecimento da integridade dos tecidos cutâneos em animais experimentais: revisão sistemática. Fisioter e Pesqui. 2012;19(3):290-6. https://doi. org/10.1590/S1809-29502012000300016

12. Gagnon D, Gibson TWG, Singh A, Linden AR, Kazienko JE, Lamarre J. An in vitro method to test the safety and efficacy of low-level laser therapy (LLLT) in the healing of a canine skin model. BMC Vet Res. 2016;12(73). https://doi. org/10.1186/s12917-016-0689-5

13. Kilík R, Lakyová L, Sabo J, Kruzliak P, Lacjaková K, Vasilenko T, Vidová M, Longauer F, Radonak J. Effect of equal daily doses achieved by different power densities of low-level laser therapy at $635 \mathrm{~nm}$ on open skin wound healing in normal and diabetic rats. Biomed Res Int. 2014;2014(269253). https://doi.org/10.1155/2014/269253

14. Rambo CS de M, Silva Jr JA, Serra AJ, Ligeiro AP, Vieira RP, Albertini R, Leal-Junior ECP, Carvalho PTC. Comparative analysis of low-level laser therapy $(660 \mathrm{~nm})$ on inflammatory biomarker expression during the skin wound-repair process in young and aged rats. Lasers Med Sci. 2014;29(5):1723-33. https://doi.org/10.1007/s10103-014-1582-8

15. Tatmatsu-Rocha JC, Ferraresi C, Hamblin MR, Maia FD, Nascimento NRF, Driusso P, Parizotto NA. Low-level laser therapy $(904 \mathrm{~nm})$ can increase collagen and reduce oxidative and nitrosative stress in diabetic wounded mouse skin. J Photochem Photobiol B Biol. 2016;164:96-102. https://doi.org/10.1016/j.jphotobiol.2016.09.017

16. de Angelis F, Kolesnikova L, Renato F, Liguori G. Fractional nonablative 1540-nm laser treatment of striae distensae in Fitzpatrick skin types II to IV: clinical and histological results. Aesthetic Surg J. 2011;31(4):411-9. https://doi. org/10.1177/1090820X11402493

17. Goldberg DJ, Marmur ES, Schmults C, Hussain M, Phelps R. Histologic and ultrastructural analysis of ultraviolet B laser and light source treatment of leukoderma in striae distensae. Dermatologic Surg. 2005;31(4):385-7. https://doi. org/10.1097/00042728-200504000-00001

18. Lee SE, Kim JH, Lee SJ, Lee JE, Kang JM, Kim YK, Bang D, Cho SB. Treatment of striae distensae using an ablative 10,600-nm carbon dioxide fractional laser: A retrospective review of 27 participants. Dermatologic Surg. 2010;36(11): 1683-90. https://doi.org/10.1111/j.1524-4725.2010.01719.x

19. Ostovari N, Saadat N, Nasiri S, Moravvej H, Toossi P. The 308-nm excimer laser in the darkening of the white lines of striae alba. J Dermatolog Treat. 2010;21(4):229-31. https://doi.org/10.3109/09546631003592044

20. Shin JU, Roh MR, Rah DK, Ae NK, Suh H, Chung KY. The effect of succinylated atelocollagen and ablative fractional resurfacing laser on striae distensae. J Dermatolog Treat. 2011;22(2):113-21. https://doi.org/10.3109/09546630903476902

21. Tay YK, Kwok C, Tan E. Non-ablative 1,450-nm diode laser treatment of striae distensae. Lasers Surg Med. 2006;38(3): 196-9. https://doi.org/10.1002/1sm.20281 
22. Ryu H-W, Kim S-A, Jung HR, Ryoo Y-W, Lee K-S, Cho J-W. Clinical improvement of striae distensae in korean patients using a combination of fractionated microneedle radiofrequency and fractional carbon dioxide laser. Dermatologic Surg. 2013;39(10):1452-8. https://doi.org/10.1111/dsu.12268

23. Yang YJ, Lee GY. Treatment of striae distensae with nonablative fractional laser versus ablative CO2 fractional laser: A randomized controlled trial. Ann Dermatol. 2011;23(4):481-9. https://doi.org/10.5021/ad.2011.23.4.481

24. Minatel DG, Enwemeka CS, França SC, Frade MAC. Phototherapy (LEDs 660/890nm) in the treatment of leg ulcers in diabetic patients: case study. An Bras Dermatol. 2009;84(3):279-83. https://doi.org/10.1590/S0365-05962009000300011

25. Rocha Júnior AM, Oliveira RG de, Farias RE, Andrade LCF de, Aarestrup FM. Modulação da proliferação fibroblástica e da resposta inflamatória pela terapia a laser de baixa intensidade no processo de reparo tecidual. An Bras Dermatol. 2006;81(2):150-6. https://doi.org/10.1590/S0365-05962006000200006

26. Guerra FDR, Vieira CP, Almeida MS, Oliveira LP, Aro AA De, Pimentel ER. LLLT improves tendon healing through increase of MMP activity and collagen synthesis. Lasers Med Sci. 2013;28(5):1281-8. https://doi.org/10.1007/s10103012-1236-7

27. Suzuki R, Takakuda K. Wound healing efficacy of a 660-nm diode laser in a rat incisional wound model. Lasers Med Sci. 2016;31(8):1683-9. https://doi.org/10.1007/s10103-016-2038-0

28. Al-Himdani S, Ud-Din S, Gilmore S, Bayat A. Striae distensae: a comprehensive review and evidence-based evaluation of prophylaxis and treatment. Br J Dermatol. 2014;170(3):527-47. https://doi.org/10.1111/bjd.12681 